\title{
Motion camouflage in three dimensions
}

\author{
P. V. Reddy, E. W. Justh, and P. S. Krishnaprasad
}

\begin{abstract}
We formulate and analyze a three-dimensional model of motion camouflage, a stealth strategy observed in nature. A high-gain feedback law for motion camouflage is formulated in which the pursuer and evader trajectories are described using natural Frenet frames (or relatively parallel adapted frames), and the corresponding natural curvatures serve as controls. The biological plausibility of the feedback law is discussed, as is its connection to missile guidance. Simulations illustrating motion camouflage are also presented. This paper builds on recent work on motion camouflage in the planar setting [8].
\end{abstract}

\section{INTRODUCTION}

Motion camouflage is a stealth strategy employed by various visual insects and animals to achieve prey capture, mating or territorial combat. In one type of motion camouflage, the predator camouflages itself against a fixed background object so that the prey observes no relative motion between the predator and the fixed object. In the other type of motion camouflage, the predator approaches the prey such that from the point of view of the prey, the predator always appears to be at the same bearing. (In this case, we say that the object against which the predator is camouflaged is the point at infinity.) For background on motion camouflage, see [8] and the references therein. Motion camouflage behavior in insects is described in [13] (based on earlier work in [4] on hoverflies) and in [9] (for dragonflies). Related themes in insect vision and flight control are also found in [14].

The essential features of motion camouflage are not limited to visual insects. Recent work on the neuroethology of insect-capture behavior in echolocating bats reveals a strategy geometrically indistinguishable from motion camouflage, referred to as the "constant absolute target direction" (CATD) strategy [5]. Because the bat under study, Eptesicus fuscus, hunts at night, there is no reason to suppose that camouflage (i.e., misleading its prey's visual system) is the bat's goal in using the CATD strategy. In this paper, we are concerned with describing in the simplest possible,

This research was supported in part by the Naval Research Laboratory under Grants No. N00173-02-1G002, N00173-03-1G001, N00173-03-1G019, and N00173-04-1G014; by the Air Force Office of Scientific Research under AFOSR Grants No. F49620-01-0415 and FA95500410130; by the Army Research Office under ODDR\&E MURI01 Program Grant No. DAAD1901-1-0465 to the Center for Communicating Networked Control Systems (through Boston University); and by NIH-NIBIB grant 1 R01 EB00475001, as part of the NSF/NIH Collaborative Research in Computational Neuroscience Program.

P.V. Reddy and P.S. Krishnaprasad are with the Institute for Systems Research and the Department of Electrical and Computer Engineering at the University of Maryland, College Park, MD 20742, USA. vishwa@umd.edu, krishna@umd.edu

E.W. Justh is with the Institute for Systems Research at the University of Maryland, College Park, MD 20742, USA. justh@umd.edu biologically plausible way how the motion camouflage or CATD strategy can be achieved using feedback control. This is a small first step toward understanding the much more difficult question of why an animal like the bat Eptesicus fuscus uses such a strategy.

What sets this work apart is the structured approach used to derive feedback laws for motion control in three dimensions. We model the pursuer (i.e., predator) and evader (i.e., prey) as point particles subject to curvature (steering) control. Although the speeds of the particles may vary, this variation is considered to result primarily from flight conditions the animal experiences - not primarily as a result of explicit speed control for purposes of achieving motion camouflage. Indeed, the feedback law we derive for motion camouflage is welldefined for constant-speed motion. However, for comparing the theoretical feedback law to the experimentally-derived bat trajectory data, it is useful to retain speed variability in the model, since speed variations on the order of 50 percent are observed as the bat maneuvers.

This focus on systematic formulation and analysis of biologically plausible feedback laws for motion camouflage is a distinguishing feature of our work. For example, in [6] motion camouflage trajectories are studied, but without explicitly providing feedback laws which give rise to them. In [1], feedback using neural networks is used to achieve motion camouflage, but our approach has the advantage of giving an explicit form and straightforward physical interpretation for the feedback control law.

In earlier work, motion camouflage in the planar setting was studied, and a feedback law to achieve motion camouflage was derived [8]. The name given to the feedback law was motion camouflage proportional guidance (MCPG). Here, we extend this work by formulating the problem in three dimensions and generalizing the feedback law to the three dimensional setting. The key is to describe the particle trajectories using natural Frenet frames [3] - the same approach demonstrated successfully in the context of formation control for constant-speed particles [7]. This formulation can also be used to describe missile guidance, specifically, pure proportional navigation guidance (PPNG) [12], [10], [11], cleanly in three dimensions.

\section{Pursuit-eVASION MODEL}

For concreteness, we consider the problem of motion camouflage in which the predator (which we refer to as the "pursuer") attempts to intercept the prey (which we refer to as the "evader") while appearing to the prey as though it is always at the same bearing (i.e., motion camouflaged against the point at infinity). The dynamics of the pursuer are given 


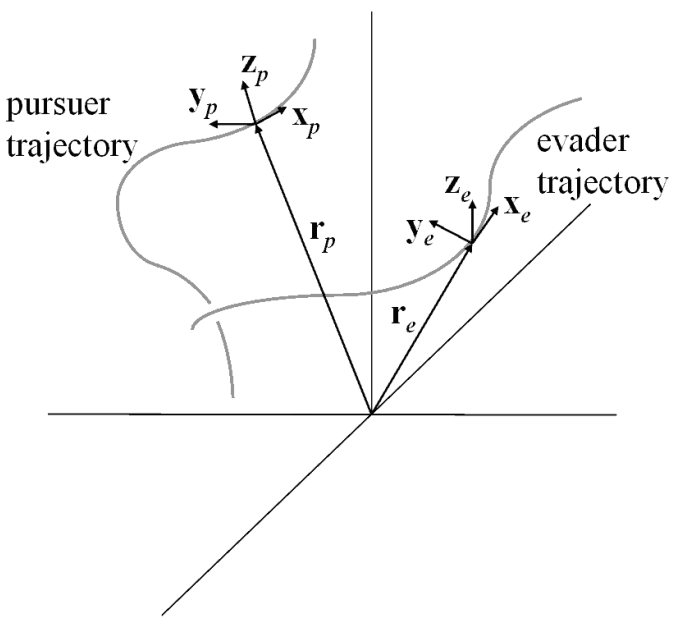

Fig. 1. Trajectories for the pursuer and evader, and their respective natural Frenet frames. The position of the pursuer is $\mathbf{r}_{p}$, and its natural Frenet frame is $\left\{\mathbf{x}_{p}, \mathbf{y}_{p}, \mathbf{z}_{p}\right\}$, where $\mathbf{x}_{p}$ is the unit tangent vector to its trajectory, and $\left\{\mathbf{y}_{p}, \mathbf{z}_{p}\right\}$ span the corresponding normal plane (and similarly for the evader). The pursuer moves with speed $\nu_{p}$, and the evader with speed $\nu_{e}$.

by

$$
\begin{aligned}
\dot{\mathbf{r}}_{p} & =\nu_{p} \mathbf{x}_{p}, \\
\dot{\mathbf{x}}_{p} & =\nu_{p}\left(\mathbf{y}_{p} u_{p}+\mathbf{z}_{p} v_{p}\right), \\
\dot{\mathbf{y}}_{p} & =-\nu_{p} \mathbf{x}_{p} u_{p}, \\
\dot{\mathbf{z}}_{p} & =-\nu_{p} \mathbf{x}_{p} v_{p},
\end{aligned}
$$

where $\mathbf{r}_{p}$ is the position of the pursuer, $\nu_{p}$ is the speed of the pursuer, $\mathbf{x}_{p}$ is the unit tangent vector to the trajectory of the pursuer, $\mathbf{y}_{p}$ and $\mathbf{z}_{p}$ span the normal plane to $\mathbf{x}_{p}$ (completing a right-handed orthonormal basis with $\mathbf{x}_{p}$ ), and the natural curvatures $u_{p}$ and $v_{p}$ are the controls for the pursuer. Similarly, the dynamics of the evader are

$$
\begin{aligned}
\dot{\mathbf{r}}_{e} & =\nu_{e} \mathbf{x}_{e}, \\
\dot{\mathbf{x}}_{e} & =\nu_{e}\left(\mathbf{y}_{e} u_{e}+\mathbf{z}_{e} v_{e}\right), \\
\dot{\mathbf{y}}_{e} & =-\nu_{e} \mathbf{x}_{e} u_{e}, \\
\dot{\mathbf{z}}_{e} & =-\nu_{e} \mathbf{x}_{e} v_{e},
\end{aligned}
$$

where $\mathbf{r}_{e}$ is the position of the evader, $\nu_{e}$ is the speed of the evader, $\mathbf{x}_{e}$ is the unit tangent vector to the trajectory of the evader, $\mathbf{y}_{e}$ and $\mathbf{z}_{e}$ span the normal plane to $\mathbf{x}_{e}$ (completing a right-handed orthonormal basis with $\mathbf{x}_{e}$ ), and the natural curvatures $u_{e}$ and $v_{e}$ are the controls for the evader. Figure 1 illustrates equations (1) and (2). Note that $\left\{\mathbf{x}_{p}, \mathbf{y}_{p}, \mathbf{z}_{p}\right\}$ and $\left\{\mathbf{x}_{e}, \mathbf{y}_{e}, \mathbf{z}_{e}\right\}$ are natural Frenet frames (also known as relatively parallel adapted frames) for the trajectories of the pursuer and evader, respectively [3].

We model the pursuer and evader as point particles, and use natural frames and curvature controls to describe their motion, because this is a simple model for which we can derive both physical intuition and concrete control laws. Flying insects and animals (also unmanned aerial vehicles) have limited maneuverability and must maintain sufficient airspeed to stay aloft, so modeling them in this way is physically reasonable, at least for some range of flight conditions.

Note that the forces supplied by the curvature controls are perpendicular to the instantaneous direction of motion, and therefore do not change the speed: these forces are gyroscopic forces. However, in (1) and (2) we do allow for the possibility of speed variations, as well.

\section{A. Characterizing motion camouflage}

Motion camouflage with respect to the point at infinity is given by [8]

$$
\mathbf{r}_{p}=\mathbf{r}_{e}+\lambda \mathbf{r}_{\infty}
$$

where $\mathbf{r}_{\infty}$ is a fixed unit vector and $\lambda$ is a time-dependent scalar (see also Section 5 of [6]).

Let

$$
\mathbf{r}=\mathbf{r}_{p}-\mathbf{r}_{e}
$$

be the vector from the evader to the pursuer. We refer to $\mathbf{r}$ as the "baseline vector," and $|\mathbf{r}|$ as the "baseline length." We restrict attention to non-collision states, i.e., $\mathbf{r} \neq 0$. In that case, the component of the pursuer velocity $\dot{\mathbf{r}}_{p}$ transverse to the base line is

$$
\dot{\mathbf{r}}_{p}-\left(\frac{\mathbf{r}}{|\mathbf{r}|} \cdot \dot{\mathbf{r}}_{p}\right) \frac{\mathbf{r}}{|\mathbf{r}|},
$$

and similarly, that of the evader is

$$
\dot{\mathbf{r}}_{e}-\left(\frac{\mathbf{r}}{|\mathbf{r}|} \cdot \dot{\mathbf{r}}_{e}\right) \frac{\mathbf{r}}{|\mathbf{r}|} .
$$

The relative transverse component is

$$
\begin{aligned}
\mathbf{w} & =\left(\dot{\mathbf{r}}_{p}-\dot{\mathbf{r}}_{e}\right)-\left(\frac{\mathbf{r}}{|\mathbf{r}|} \cdot\left(\dot{\mathbf{r}}_{p}-\dot{\mathbf{r}}_{e}\right)\right) \frac{\mathbf{r}}{|\mathbf{r}|} \\
& =\dot{\mathbf{r}}-\left(\frac{\mathbf{r}}{|\mathbf{r}|} \cdot \dot{\mathbf{r}}\right) \frac{\mathbf{r}}{|\mathbf{r}|}
\end{aligned}
$$

Lemma (Infinitesimal characterization of motion camouflage): The pursuit-evasion system (1), (2) is in a state of motion camouflage without collision on an interval iff $\mathbf{w}=\mathbf{0}$ on that interval.

Proof: $(\Longrightarrow)$ Suppose motion camouflage holds. Thus

$$
\mathbf{r}(t)=\lambda(t) \mathbf{r}_{\infty}, \quad t \in[0, T]
$$

Differentiating, $\dot{\mathbf{r}}=\dot{\lambda} \mathbf{r}_{\infty}$. Hence,

$$
\begin{aligned}
\mathbf{w} & =\dot{\mathbf{r}}-\left(\frac{\mathbf{r}}{|\mathbf{r}|} \cdot \dot{\mathbf{r}}\right) \frac{\mathbf{r}}{|\mathbf{r}|} \\
& =\dot{\lambda} \mathbf{r}_{\infty}-\left(\frac{\lambda}{|\lambda|} \mathbf{r}_{\infty} \cdot \dot{\lambda} \mathbf{r}_{\infty}\right) \frac{\lambda}{|\lambda|} \mathbf{r}_{\infty} \\
& =\mathbf{0} \text { on }[0, T] .
\end{aligned}
$$

$(\Longleftarrow)$ Suppose $\mathbf{w}=\mathbf{0}$ on $[0, T]$. Thus

$$
\dot{\mathbf{r}}=\left(\frac{\mathbf{r}}{|\mathbf{r}|} \cdot \dot{\mathbf{r}}\right) \frac{\mathbf{r}}{|\mathbf{r}|} \triangleq \xi \mathbf{r},
$$




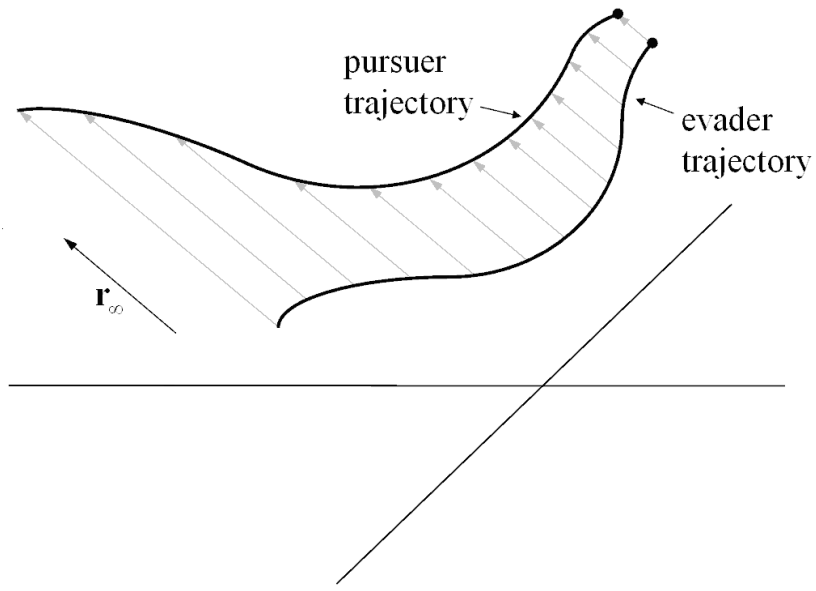

Fig. 2. Pursuer and evader trajectories in a state of motion camouflage with respect to the point at infinity, i.e., satisfying $\mathbf{r}_{p}-\mathbf{r}_{e}=\lambda \mathbf{r}_{\infty}$, where $r_{\infty}$ is fixed and $\lambda$ varies with time. The light gray vectors are baseline vectors at different instants of time: note that they are all parallel to one another.

so that

$$
\begin{aligned}
\mathbf{r}(t) & =\exp \left(\int_{0}^{t} \xi(\sigma) d \sigma\right) \mathbf{r}(0) \\
& =|\mathbf{r}(0)| \exp \left(\int_{0}^{t} \xi(\sigma) d \sigma\right) \frac{\mathbf{r}(0)}{|\mathbf{r}(0)|} \\
& =\lambda(t) \mathbf{r}_{\infty},
\end{aligned}
$$

where $\quad \mathbf{r}_{\infty}=\mathbf{r}(0) /|\mathbf{r}(0)|$ and $\lambda(t)=$ $|\mathbf{r}(0)| \exp \left(\int_{0}^{t} \xi(\sigma) d \sigma\right)$.

Remark: The above Lemma and its proof are identical to the corresponding Lemma and proof in [8], but with the vectors interpreted as three-dimensional rather than planar vectors.

Figure 2 illustrates the pursuer and evader in a state of motion camouflage with respect to the point at infinity.

\section{B. Measuring departure from motion camouflage}

Consider the ratio

$$
\Gamma(t)=\frac{\frac{d}{d t}|\mathbf{r}|}{\left|\frac{d \mathbf{r}}{d t}\right|},
$$

which compares the rate of change of the baseline length to the absolute rate of change of the baseline vector [8]. If the baseline experiences pure lengthening, then the ratio assumes its maximum value, $\Gamma(t)=1$. If the baseline experiences pure shortening, then the ratio assumes its minimum value, $\Gamma(t)=-1$. If the baseline experiences pure rotation, but remains the same length, then $\Gamma(t)=0$. Noting that

$$
\frac{d}{d t}|\mathbf{r}|=\frac{\mathbf{r}}{|\mathbf{r}|} \cdot \dot{\mathbf{r}}
$$

we see that $\Gamma(t)$ may alternatively be written as

$$
\Gamma(t)=\frac{\mathbf{r}}{|\mathbf{r}|} \cdot \frac{\dot{\mathbf{r}}}{|\dot{\mathbf{r}}|} .
$$

Thus, $\Gamma(t)$ is the dot product of two unit vectors: one in the direction of $\mathbf{r}$, and the other in the direction of $\dot{\mathbf{r}}$.

From

$$
\begin{aligned}
|\mathbf{w}|^{2} & =|\dot{\mathbf{r}}|^{2}-2\left(\frac{\mathbf{r}}{|\mathbf{r}|} \cdot \dot{\mathbf{r}}\right)^{2}+\left(\frac{\mathbf{r}}{|\mathbf{r}|} \cdot \dot{\mathbf{r}}\right)^{2} \\
& =|\dot{\mathbf{r}}|^{2}\left(1-\Gamma^{2}\right),
\end{aligned}
$$

it follows that $\left(1-\Gamma^{2}\right)$ is a measure of departure from motion camouflage.

\section{FEEDBACK LAW FOR MOTION CAMOUFLAGE}

Using the planar setting as a guide, the curvature controls to achieve motion camouflage in three dimensions can be systematically derived. Indeed, this is a major advantage of representing trajectories using natural Frenet frames. However, for ease of exposition, we instead begin by presenting the control law in an intuitively appealing and biologically plausible form, followed by the calculations demonstrating its effectiveness.

\section{A. Feedback law and interpretation}

Using the BAC-CAB identity, $\tilde{\mathbf{a}} \times(\tilde{\mathbf{b}} \times \tilde{\mathbf{c}})=\tilde{\mathbf{b}}(\tilde{\mathbf{a}} \cdot \tilde{\mathbf{c}})-$ $\tilde{\mathbf{c}}(\tilde{\mathbf{a}} \cdot \tilde{\mathbf{b}})$, for arbitrary vectors $\tilde{\mathbf{a}}, \tilde{\mathbf{b}}, \tilde{\mathbf{c}}$, we observe that

$$
\begin{aligned}
\mathbf{w}=\dot{\mathbf{r}}\left(\frac{\mathbf{r}}{|\mathbf{r}|} \cdot \frac{\mathbf{r}}{|\mathbf{r}|}\right)-\frac{\mathbf{r}}{|\mathbf{r}|}\left(\frac{\mathbf{r}}{|\mathbf{r}|} \cdot \dot{\mathbf{r}}\right)=\frac{\mathbf{r}}{|\mathbf{r}|} \times\left(\dot{\mathbf{r}} \times \frac{\mathbf{r}}{|\mathbf{r}|}\right), \\
\mathbf{w} \times \frac{\mathbf{r}}{|\mathbf{r}|}=\left[\frac{\mathbf{r}}{|\mathbf{r}|} \times\left(\dot{\mathbf{r}} \times \frac{\mathbf{r}}{|\mathbf{r}|}\right)\right] \times \frac{\mathbf{r}}{|\mathbf{r}|} \\
=-\frac{\mathbf{r}}{|\mathbf{r}|}\left[\left(\dot{\mathbf{r}} \times \frac{\mathbf{r}}{|\mathbf{r}|}\right) \cdot \frac{\mathbf{r}}{|\mathbf{r}|}\right]+\left(\dot{\mathbf{r}} \times \frac{\mathbf{r}}{|\mathbf{r}|}\right) \\
=\dot{\mathbf{r}} \times \frac{\mathbf{r}}{|\mathbf{r}|},
\end{aligned}
$$

and we conclude from (15) that $(\dot{\mathbf{r}} \times \mathbf{r} /|\mathbf{r}|)$ is a biologically plausible quantity to appear in a feedback law, since it only requires sensing $\mathbf{w}$ and $\mathbf{r} /|\mathbf{r}|$.

The quantity $(\dot{\mathbf{r}} \times \mathbf{r} /|\mathbf{r}|)$ can be interpreted in terms of an angular-velocity-like quantity. From the point of view of the pursuer, consider an extensible rod connecting the pursuer and evader positions. The motion of the evader (relative to the pursuer) contributes to change in the length of this rod, as well as to angular velocity of the rod (viewed from the pursuer - see figure 3). The transverse component of the velocity of the evader (viewed from the pursuer) is simply

$$
\begin{gathered}
\left(\dot{\mathbf{r}}_{e}-\dot{\mathbf{r}}_{p}\right)-\left[\left(\dot{\mathbf{r}}_{e}-\dot{\mathbf{r}}_{p}\right) \cdot \frac{\mathbf{r}_{e}-\mathbf{r}_{p}}{\left|\mathbf{r}_{e}-\mathbf{r}_{p}\right|}\right] \frac{\mathbf{r}_{e}-\mathbf{r}_{p}}{\left|\mathbf{r}_{e}-\mathbf{r}_{p}\right|} \\
\quad=-\dot{\mathbf{r}}-\left[-\dot{\mathbf{r}} \cdot\left(-\frac{\mathbf{r}}{|\mathbf{r}|}\right)\right]\left(-\frac{\mathbf{r}}{|\mathbf{r}|}\right) \\
\quad=-\mathbf{w},
\end{gathered}
$$

which can also be expressed as

$$
-\mathbf{w}=\omega \times(-\mathbf{r}),
$$

where $\boldsymbol{\omega}$ is the corresponding angular velocity of the rod. From (14) and 17) we conclude that

$$
\frac{\mathbf{r}}{|\mathbf{r}|} \times\left(\dot{\mathbf{r}} \times \frac{\mathbf{r}}{|\mathbf{r}|}\right)=\left(\frac{\mathbf{r}}{|\mathbf{r}|^{2}} \times \dot{\mathbf{r}}\right) \times \mathbf{r}=\boldsymbol{\omega} \times \mathbf{r}
$$




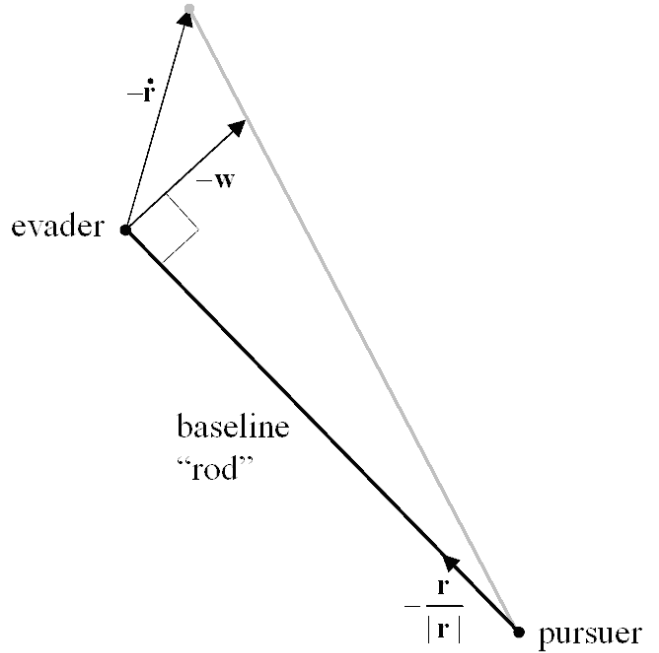

Fig. 3. Motion of the rod connecting the evader to the pursuer, from the point of view of the pursuer. The angular velocity of the rod, $\boldsymbol{\omega}$, is a vector pointing into the page.

and hence

$$
\omega=\frac{\mathbf{r}}{|\mathbf{r}|^{2}} \times \dot{\mathbf{r}}
$$

Thus, the quantity $(\dot{\mathbf{r}} \times \mathbf{r} /|\mathbf{r}|)$ is simply $-\boldsymbol{\omega}$ scaled by $|\mathbf{r}|$.

For convenience in the calculations below, we define

$$
\mathbf{a}=\mathbf{x}_{p} \times\left(\dot{\mathbf{r}} \times \frac{\mathbf{r}}{|\mathbf{r}|}\right)
$$

and express the feedback law as

$$
\begin{aligned}
& u_{p}=\mu\left(\mathbf{a} \cdot \mathbf{y}_{p}\right), \\
& v_{p}=\mu\left(\mathbf{a} \cdot \mathbf{z}_{p}\right),
\end{aligned}
$$

where $\mu>0$ is a constant feedback gain. The quantity $\mu \nu_{p}^{2}$ a can then be interpreted as the lateral component of the acceleration vector of the pursuer. Consistent with the fact that $u_{p}$ and $v_{p}$ can only change the direction of the pursuer's motion and not its speed, we note that $\mu \nu_{p}^{2} \mathbf{a}$ is transverse to the direction of motion of the pursuer, $\mathbf{x}_{\tilde{\tau}}$ : i.e., $\mathbf{a} \cdot \mathbf{x}_{p}=0$.

Using the formula $\tilde{\mathbf{a}} \cdot(\tilde{\mathbf{b}} \times \tilde{\mathbf{c}})=\tilde{\mathbf{b}} \cdot(\tilde{\mathbf{c}} \times \tilde{\mathbf{a}})$ for the scalar triple product, where $\tilde{\mathbf{a}}, \tilde{\mathbf{b}}, \tilde{\mathbf{c}}$ are arbitrary vectors, we compute

$$
\begin{aligned}
u_{p} & =\mu\left[\mathbf{x}_{p} \times\left(\dot{\mathbf{r}} \times \frac{\mathbf{r}}{|\mathbf{r}|}\right)\right] \cdot \mathbf{y}_{p} \\
& =\mu\left[\left(\dot{\mathbf{r}} \times \frac{\mathbf{r}}{|\mathbf{r}|}\right) \cdot\left(\mathbf{y}_{p} \times \mathbf{x}_{p}\right)\right] \\
& =-\mu\left[\left(\dot{\mathbf{r}} \times \frac{\mathbf{r}}{|\mathbf{r}|}\right) \cdot \mathbf{z}_{p}\right],
\end{aligned}
$$

and similarly,

$$
v_{p}=\mu\left[\left(\dot{\mathbf{r}} \times \frac{\mathbf{r}}{|\mathbf{r}|}\right) \cdot \mathbf{y}_{p}\right] .
$$

Remark: It is easy to see that in the planar setting, we recover the planar steering law for motion camouflage presented in [8]. If $\mathbf{x}_{p}, \mathbf{x}_{e}$, and $\mathbf{r}$ all lie in the same plane, then $\dot{\mathbf{r}}$ also lies in that plane, and (22) becomes

$$
u_{p}=-\mu\left[\left(\dot{\mathbf{r}} \times \frac{\mathbf{r}}{|\mathbf{r}|}\right) \cdot \mathbf{z}_{p}\right]=-\mu\left(\frac{\mathbf{r}}{|\mathbf{r}|} \cdot \dot{\mathbf{r}}^{\perp}\right),
$$

where the notion $\mathbf{q}^{\perp}$ represents the vector $\mathbf{q}$ rotated counterclockwise in the plane by $\pi / 2$. Furthermore, without loss of generality, we identify $\mathbf{y}_{p}$ with $\mathbf{x}_{p}^{\perp}$, and $\mathbf{z}_{p}$ with the unit vector perpendicular to the plane of motion.

\section{B. Behavior of $\Gamma$ under the feedback law}

Differentiating $\Gamma$ along trajectories of (1) and (2) gives

$$
\begin{aligned}
\dot{\Gamma} & =\left(\frac{\dot{\mathbf{r}} \cdot \dot{\mathbf{r}}+\mathbf{r} \cdot \ddot{\mathbf{r}}}{|\mathbf{r} \| \dot{\mathbf{r}}|}\right)-\left(\frac{\mathbf{r} \cdot \dot{\mathbf{r}}}{|\dot{\mathbf{r}}|}\right)\left(\frac{\mathbf{r} \cdot \dot{\mathbf{r}}}{|\mathbf{r}|^{3}}\right)-\left(\frac{\mathbf{r} \cdot \dot{\mathbf{r}}}{|\mathbf{r}|}\right)\left(\frac{\dot{\mathbf{r}} \cdot \ddot{\mathbf{r}}}{|\dot{\mathbf{r}}|^{3}}\right) \\
& =\frac{|\dot{\mathbf{r}}|}{|\mathbf{r}|}\left[1-\left(\frac{\mathbf{r}}{|\mathbf{r}|} \cdot \frac{\dot{\mathbf{r}}}{|\dot{\mathbf{r}}|}\right)^{2}\right]+\frac{1}{|\dot{\mathbf{r}}|}\left[\frac{\mathbf{r}}{|\mathbf{r}|}-\left(\frac{\mathbf{r}}{|\mathbf{r}|} \cdot \frac{\dot{\mathbf{r}}}{|\dot{\mathbf{r}}|}\right) \frac{\dot{\mathbf{r}}}{|\dot{\mathbf{r}}|}\right] \cdot \ddot{\mathbf{r}} .
\end{aligned}
$$

We also have

$$
\begin{aligned}
\ddot{\mathbf{r}} & =\dot{\nu}_{p} \mathbf{x}_{p}-\dot{\nu}_{e} \mathbf{x}_{e}+\nu_{p} \dot{\mathbf{x}}_{p}-\nu_{e} \dot{\mathbf{x}}_{e} \\
& =\dot{\nu}_{p} \mathbf{x}_{p}-\dot{\nu}_{e} \mathbf{x}_{e}+\nu_{p}^{2}\left(\mathbf{y}_{p} u_{p}+\mathbf{z}_{p} v_{p}\right)-\nu_{e}^{2}\left(\mathbf{y}_{e} u_{e}+\mathbf{z}_{e} v_{e}\right)
\end{aligned}
$$

If we define

$$
\mathbf{b}=\frac{1}{|\dot{\mathbf{r}}|}\left[\frac{\mathbf{r}}{|\mathbf{r}|}-\left(\frac{\mathbf{r}}{|\mathbf{r}|} \cdot \frac{\dot{\mathbf{r}}}{|\dot{\mathbf{r}}|}\right) \frac{\dot{\mathbf{r}}}{|\dot{\mathbf{r}}|}\right]
$$

then

$$
\begin{aligned}
\mathbf{b} \cdot \ddot{\mathbf{r}}= & \dot{\nu}_{p}\left(\mathbf{b} \cdot \mathbf{x}_{p}\right)-\dot{\nu}_{e}\left(\mathbf{b} \cdot \mathbf{x}_{e}\right) \\
& +\nu_{p}^{2}\left[\left(\mathbf{b} \cdot \mathbf{y}_{p}\right) u_{p}+\left(\mathbf{b} \cdot \mathbf{z}_{p}\right) v_{p}\right] \\
& -\nu_{e}^{2}\left[\left(\mathbf{b} \cdot \mathbf{y}_{e}\right) u_{e}+\left(\mathbf{b} \cdot \mathbf{z}_{e}\right) v_{e}\right]
\end{aligned}
$$

and the only term of $\dot{\Gamma}$ into which the controls $u_{p}$ and $v_{p}$ explicitly enter is

$$
\nu_{p}^{2}\left[\left(\mathbf{b} \cdot \mathbf{y}_{p}\right) u_{p}+\left(\mathbf{b} \cdot \mathbf{z}_{p}\right) v_{p}\right] .
$$

Using (20) and 21,

$$
\begin{aligned}
\nu_{p}^{2}\left[\left(\mathbf{b} \cdot \mathbf{y}_{p}\right)\right. & \left.u_{p}+\left(\mathbf{b} \cdot \mathbf{z}_{p}\right) v_{p}\right] \\
& =\mu \nu_{p}^{2}\left[\left(\mathbf{b} \cdot \mathbf{y}_{p}\right)\left(\mathbf{a} \cdot \mathbf{y}_{p}\right)+\left(\mathbf{b} \cdot \mathbf{z}_{p}\right)\left(\mathbf{a} \cdot \mathbf{z}_{p}\right)\right] \\
& =\mu \nu_{p}^{2}\left[(\mathbf{b} \cdot \mathbf{a})-\left(\mathbf{b} \cdot \mathbf{x}_{p}\right)\left(\mathbf{a} \cdot \mathbf{x}_{p}\right)\right] \\
& =\mu \nu_{p}^{2}(\mathbf{b} \cdot \mathbf{a})
\end{aligned}
$$

where we have also used the identity

$$
\mathbf{a} \cdot \mathbf{b}=\left(\mathbf{a} \cdot \mathbf{x}_{p}\right)\left(\mathbf{b} \cdot \mathbf{x}_{p}\right)+\left(\mathbf{a} \cdot \mathbf{y}_{p}\right)\left(\mathbf{b} \cdot \mathbf{y}_{p}\right)+\left(\mathbf{a} \cdot \mathbf{z}_{p}\right)\left(\mathbf{b} \cdot \mathbf{z}_{p}\right),
$$

and $\mathbf{a} \cdot \mathbf{x}_{p}=0$.

Using the BAC-CAB identity, we observe that

$$
\mathbf{b}=\frac{1}{|\dot{\mathbf{r}}|}\left[\frac{\dot{\mathbf{r}}}{|\dot{\mathbf{r}}|} \times\left(\frac{\mathbf{r}}{|\mathbf{r}|} \times \frac{\dot{\mathbf{r}}}{|\dot{\mathbf{r}}|}\right)\right]=-\frac{1}{|\dot{\mathbf{r}}|^{3}}\left[\dot{\mathbf{r}} \times\left(\dot{\mathbf{r}} \times \frac{\mathbf{r}}{|\mathbf{r}|}\right)\right],
$$

so that

$$
\mathbf{b} \cdot \mathbf{a}=-\frac{1}{|\dot{\mathbf{r}}|^{3}}\left[\dot{\mathbf{r}} \times\left(\dot{\mathbf{r}} \times \frac{\mathbf{r}}{|\mathbf{r}|}\right)\right] \cdot\left[\mathbf{x}_{p} \times\left(\dot{\mathbf{r}} \times \frac{\mathbf{r}}{|\mathbf{r}|}\right)\right] .
$$


Using the identity $(\tilde{\mathbf{a}} \times \tilde{\mathbf{b}}) \cdot(\tilde{\mathbf{c}} \times \tilde{\mathbf{d}})=(\tilde{\mathbf{a}} \cdot \tilde{\mathbf{c}})(\tilde{\mathbf{b}} \cdot \tilde{\mathbf{d}})-(\tilde{\mathbf{a}} \cdot \tilde{\mathbf{d}})(\tilde{\mathbf{b}} \cdot \tilde{\mathbf{c}})$, for arbitrary vectors $\tilde{\mathbf{a}}, \tilde{\mathbf{b}}, \tilde{\mathbf{c}}, \tilde{\mathbf{d}}$, and

$$
\left|\dot{\mathbf{r}} \times \frac{\mathbf{r}}{|\mathbf{r}|}\right|^{2}=|\dot{\mathbf{r}}|^{2}\left(1-\Gamma^{2}\right),
$$

we compute

$$
\begin{aligned}
\mathbf{b} \cdot \mathbf{a}=- & \frac{1}{|\dot{\mathbf{r}}|}\left(\dot{\mathbf{r}} \cdot \mathbf{x}_{p}\right)\left(1-\Gamma^{2}\right) \\
& +\frac{1}{|\dot{\mathbf{r}}|^{3}}\left[\dot{\mathbf{r}} \cdot\left(\dot{\mathbf{r}} \times \frac{\mathbf{r}}{|\mathbf{r}|}\right)\right]\left[\left(\dot{\mathbf{r}} \times \frac{\mathbf{r}}{|\mathbf{r}|}\right) \cdot \mathbf{x}_{p}\right] \\
= & -\left(1-\Gamma^{2}\right)\left(\frac{\dot{\mathbf{r}}}{|\dot{\mathbf{r}}|} \cdot \mathbf{x}_{p}\right) .
\end{aligned}
$$

Remark: For the foregoing calculations to make sense, we require $|\mathbf{r}|>0$ and $|\dot{\mathbf{r}}|>0$. The condition $|\mathbf{r}|>0$ is a non-collision condition, and does not pose any difficulty for us because our analysis of approach to the state of motion camouflage takes place away from the collision state. Later, we will impose hypotheses that also ensure $|\dot{\mathbf{r}}|>0$ for all time. (Note that in the constant-speed setting, $0<\nu_{e} / \nu_{p}<1$ is sufficient to ensure $|\dot{\mathbf{r}}|>0$ [8].)

Remark: Provided $\nu_{p}>\nu_{e}$, we have

$$
\left(\frac{\dot{\mathbf{r}}}{|\dot{\mathbf{r}}|} \cdot \mathbf{x}_{p}\right)=\frac{1}{|\dot{\mathbf{r}}|}\left[\nu_{p}-\nu_{e}\left(\mathbf{x}_{p} \cdot \mathbf{x}_{e}\right)\right]>0
$$

so that

$$
\mathbf{b} \cdot \mathbf{a} \leq 0
$$

and therefore the only term in $\dot{\Gamma}$ explicitly involving the controls $u_{p}$ and $v_{p}$ satisfies

$$
\nu_{p}^{2}\left[\left(\mathbf{b} \cdot \mathbf{y}_{p}\right) u_{p}+\left(\mathbf{b} \cdot \mathbf{z}_{p}\right) v_{p}\right] \leq 0 .
$$

To summarize, (25) becomes

$$
\begin{aligned}
\dot{\Gamma}= & -\left(1-\Gamma^{2}\right)\left[\frac{\mu \nu_{p}^{2}}{|\dot{\mathbf{r}}|}\left(\nu_{p}-\nu_{e}\left(\mathbf{x}_{p} \cdot \mathbf{x}_{e}\right)\right)-\frac{|\dot{\mathbf{r}}|}{|\mathbf{r}|}\right] \\
& +\dot{\nu}_{p}\left(\mathbf{b} \cdot \mathbf{x}_{p}\right)-\dot{\nu}_{e}\left(\mathbf{b} \cdot \mathbf{x}_{e}\right) \\
& -\nu_{e}^{2}\left[\left(\mathbf{b} \cdot \mathbf{y}_{e}\right) u_{e}+\left(\mathbf{b} \cdot \mathbf{z}_{e}\right) v_{e}\right] .
\end{aligned}
$$

Noting that

$$
|\mathbf{b}|^{2}=\frac{1}{|\dot{\mathbf{r}}|^{2}}\left(1-\Gamma^{2}\right)
$$

we see that

$$
\begin{aligned}
& \left|\nu_{e}^{2}\left[\left(\mathbf{b} \cdot \mathbf{y}_{e}\right) u_{e}+\left(\mathbf{b} \cdot \mathbf{z}_{e}\right) v_{e}\right]\right| \\
& \quad \leq \frac{\nu_{e}^{2}}{|\dot{\mathbf{r}}|} \sqrt{1-\Gamma^{2}} \max \left(\sqrt{u_{e}^{2}+v_{e}^{2}}\right)
\end{aligned}
$$

where $\max \left(\sqrt{u_{e}^{2}+v_{e}^{2}}\right)$ is an a priori bound on the maximum absolute curvature of the evader trajectory. Similarly,

$$
\begin{aligned}
\mid \dot{\nu}_{p}(\mathbf{b} & \left.\cdot \mathbf{x}_{p}\right)-\dot{\nu}_{e}\left(\mathbf{b} \cdot \mathbf{x}_{e}\right) \mid \\
& \leq \frac{1}{|\dot{\mathbf{r}}|} \sqrt{1-\Gamma^{2}}\left(\left|\dot{\nu}_{p}\right|+\left|\dot{\nu}_{e}\right|\right) \\
& \leq \frac{1}{|\dot{\mathbf{r}}|} \sqrt{1-\Gamma^{2}}\left(\alpha_{p}+\alpha_{e}\right)
\end{aligned}
$$

where $\alpha_{p}$ is an upper bound on $\left|\dot{\nu}_{p}\right|$, and $\alpha_{e}$ is an upper bound on $\left|\dot{\nu}_{e}\right|$. From (39) we then conclude

$$
\begin{aligned}
\dot{\Gamma} \leq & \left(1-\Gamma^{2}\right)\left[\frac{\mu \nu_{p}^{2}}{|\dot{\mathbf{r}}|}\left(\nu_{p}-\nu_{e}\left(\mathbf{x}_{p} \cdot \mathbf{x}_{e}\right)\right)-\frac{|\dot{\mathbf{r}}|}{|\mathbf{r}|}\right] \\
& +\frac{1}{|\dot{\mathbf{r}}|} \sqrt{1-\Gamma^{2}}\left[\alpha_{p}+\alpha_{e}+\nu_{e}^{2} \max \left(\sqrt{u_{e}^{2}+v_{e}^{2}}\right)\right] .
\end{aligned}
$$

\section{Bounds and estimates}

Having bounded $\dot{\Gamma}$ as in (43), we proceed in analogy with the planar setting [8]. We hypothesize that a constant $\nu_{\max }$ exists such that

$$
\frac{\nu_{e}}{\nu_{p}} \leq \nu_{\max }<1,
$$

for all time. We also assume that constants $\nu_{p}^{\text {low }}, \nu_{p}^{\text {high }}, \nu_{e}^{\text {low }}$, and $\nu_{e}^{\text {high }}$ exist such that

$$
\begin{aligned}
& 0<\nu_{p}^{\text {low }} \leq \nu_{p} \leq \nu_{p}^{\text {high }}<\infty \\
& 0<\nu_{e}^{\text {low }} \leq \nu_{e} \leq \nu_{e}^{\text {high }}<\infty
\end{aligned}
$$

for all time, and observe that

$$
0<\nu_{p}^{\text {low }}\left(1-\nu_{\max }\right) \leq|\dot{\mathbf{r}}| \leq \nu_{p}^{\text {high }}\left(1+\nu_{\max }\right) .
$$

We define the constant $c_{1}>0$ as

$$
c_{1}=\frac{\left[\alpha_{p}+\alpha_{e}+\left(\nu_{e}^{\text {high }}\right)^{2} \max \left(\sqrt{u_{e}^{2}+v_{e}^{2}}\right)\right]}{\nu_{p}^{\text {low }}\left(1-\nu_{\max }\right)} .
$$

Given $\mu>0$ sufficiently large and $r_{o}>0$, we define $c_{0}>0$ by

$$
c_{0}=\left(\frac{\left(\nu_{p}^{l o w}\right)^{3}\left(1-\nu_{\max }\right)}{\nu_{p}^{\text {high }}\left(1+\nu_{\max }\right)}\right) \mu-\frac{\nu_{p}^{\text {high }}\left(1+\nu_{\max }\right)}{r_{o}},
$$

so that

$$
\mu=\left(\frac{\nu_{p}^{\text {high }}\left(1+\nu_{\max }\right)}{\left(\nu_{p}^{\text {low }}\right)^{3}\left(1-\nu_{\max }\right)}\right)\left(\frac{\nu_{p}^{\text {high }}\left(1+\nu_{\max }\right)}{r_{o}}+c_{0}\right),
$$

and hence

$$
\mu \geq\left(\frac{\nu_{p}^{\text {high }}\left(1+\nu_{\max }\right)}{\left(\nu_{p}^{\text {low }}\right)^{3}\left(1-\nu_{\max }\right)}\right)\left(\frac{\nu_{p}^{\text {high }}\left(1+\nu_{\max }\right)}{|\mathbf{r}|}+c_{0}\right),
$$


$\forall|\mathbf{r}| \geq r_{o}$. Thus, for $|\mathbf{r}| \geq r_{o}$, 43 becomes

$$
\begin{aligned}
\dot{\Gamma} \leq- & \left(1-\Gamma^{2}\right)\left[\left(\frac{\nu_{p}^{\text {high }}\left(1+\nu_{\max }\right)}{\left(\nu_{p}^{\text {low }}\right)^{3}\left(1-\nu_{\max }\right)}\right)\left(\frac{\nu_{p}^{\text {high }}\left(1+\nu_{\max }\right)}{|\mathbf{r}|}+c_{0}\right)\right. \\
& \left.\times\left(\frac{\left(\nu_{p}^{\text {low }}\right)^{3}\left(1-\nu_{\max }\right)}{\nu_{p}^{\text {high }}\left(1+\nu_{\max }\right)}\right)-\frac{\nu_{p}^{\text {high }}\left(1+\nu_{\max }\right)}{|\mathbf{r}|}\right] \\
& +\left(\sqrt{1-\Gamma^{2}}\right) c_{1} \\
=- & \left(1-\Gamma^{2}\right) c_{0}+\left(\sqrt{1-\Gamma^{2}}\right) c_{1} .
\end{aligned}
$$

Suppose that given $0<\epsilon<<1$, we take $\mu>0$ sufficiently large so that there exists $c_{0}$ satisfying $c_{0} \geq 2 c_{1} / \sqrt{\epsilon}$. Then for $\left(1-\Gamma^{2}\right)>\epsilon$,

$$
\begin{aligned}
\dot{\Gamma} & \leq-\left(1-\Gamma^{2}\right) c_{0}+\left(\sqrt{1-\Gamma^{2}}\right) c_{1} \\
& =-\left(1-\Gamma^{2}\right)\left(c_{0}-\frac{c_{1}}{\sqrt{1-\Gamma^{2}}}\right) \\
& \leq-\left(1-\Gamma^{2}\right)\left(c_{0}-\frac{c_{1}}{\sqrt{\epsilon}}\right) \\
& =-\left(1-\Gamma^{2}\right) c_{2},
\end{aligned}
$$

where

$$
c_{2}=c_{0}-\frac{c_{1}}{\sqrt{\epsilon}}>0
$$

Remark: There are two possibilities for

$$
\left(1-\Gamma^{2}\right) \leq \epsilon .
$$

The state we seek to drive the system toward has $\Gamma \approx-1$; however, 55 can also be satisfied for $\Gamma \approx 1$. (Recall that $-1 \leq \Gamma \leq 1$.) There is always a set of initial conditions such that 55 is satisfied with $\Gamma \approx 1$. We can address this issue as follows: let $\epsilon_{o}>0$ denote how close to -1 we wish to drive $\Gamma$, and let $\Gamma_{0}=\Gamma(0)$ denote the initial value of $\Gamma$. Take

$$
\epsilon=\min \left(\epsilon_{o}, 1-\Gamma_{0}^{2}\right),
$$

so that (53) with 54 applies from time $t=0$.

From (53), we can write

$$
\frac{d \Gamma}{1-\Gamma^{2}} \leq-c_{2} d t
$$

which, integrating both sides, leads to

$$
\int_{\Gamma_{0}}^{\Gamma} \frac{d \tilde{\Gamma}}{1-\tilde{\Gamma}^{2}} \leq-c_{2} \int_{0}^{t} d \tilde{t}=-c_{2} t
$$

where $\Gamma_{0}=\Gamma(t=0)$. Noting that

$$
\int_{\Gamma_{0}}^{\Gamma} \frac{d \tilde{\Gamma}}{1-\tilde{\Gamma}^{2}}=\int_{\Gamma_{0}}^{\Gamma} d\left(\tanh ^{-1} \tilde{\Gamma}\right)=\tanh ^{-1} \Gamma-\tanh ^{-1} \Gamma_{0}
$$

we see that for $|\mathbf{r}| \geq r_{o}$, 53 implies

$$
\Gamma(t) \leq \tanh \left(\tanh ^{-1} \Gamma_{0}-c_{2} t\right),
$$

where we have used the fact that $\tanh ^{-1}(\cdot)$ is a monotone increasing function.
Now we consider estimating how long $|\mathbf{r}| \geq r_{o}$, which in turn determines how large $t$ can become in inequality (60), and hence how close to -1 will $\Gamma(t)$ be driven. From (11) and (12) we have

$$
\frac{d}{d t}|\mathbf{r}|=\Gamma(t)|\dot{\mathbf{r}}|
$$

which from 47 and $|\Gamma(t)| \leq 1, \forall t$, implies

$$
\frac{d}{d t}|\mathbf{r}| \geq-|\Gamma(t)| \nu_{p}^{h i g h}\left(1+\nu_{\max }\right) \geq-\nu_{p}^{h i g h}\left(1+\nu_{\max }\right) \text {. }
$$

From (62), we conclude that

$$
|\mathbf{r}(t)| \geq|\mathbf{r}(0)|-\nu_{p}^{\text {high }}\left(1+\nu_{\max }\right) t, \quad \forall t \geq 0,
$$

and, more to the point,

$$
|\mathbf{r}(t)| \geq r_{o}, \quad \forall t \leq \frac{|\mathbf{r}(0)|-r_{o}}{\nu_{p}^{\text {high }}\left(1+\nu_{\max }\right)}
$$

For 64 to be meaningful for the problem at hand, we assume that $|\mathbf{r}(0)|>r_{o}$. Then defining

$$
T=\frac{|\mathbf{r}(0)|-r_{o}}{\nu_{p}^{\text {high }}\left(1+\nu_{\max }\right)}>0
$$

to be the minimum interval of time over which we can guarantee that $\dot{\Gamma} \leq 0$, we conclude that

$$
\Gamma(T) \leq \tanh \left(\tanh ^{-1} \Gamma_{0}-c_{2} T\right) .
$$

From (66), we see that by choosing $c_{2}$ sufficiently large (which can be accomplished by choosing $c_{0} \geq 2 c_{1} / \sqrt{\epsilon}$ sufficiently large), we can force $\Gamma(T) \leq-1+\epsilon$. Noting that

$$
\tanh (x) \leq-1+\epsilon \Longleftrightarrow x \leq \frac{1}{2} \ln \left(\frac{\epsilon}{2-\epsilon}\right),
$$

for $0<\epsilon<<1$, we see that

$$
\Gamma(T) \leq-1+\epsilon \Longleftrightarrow \tanh ^{-1} \Gamma_{0}-c_{2} T \leq \frac{1}{2} \ln \left(\frac{\epsilon}{2-\epsilon}\right) .
$$

Thus, if $c_{0} \geq 2 c_{1} / \sqrt{\epsilon}$ is taken to be sufficiently large that

$$
c_{2} \geq \nu_{p}^{\text {high }}\left(1+\nu_{\max }\right) \frac{\tanh ^{-1} \Gamma_{0}-\frac{1}{2} \ln \left(\frac{\epsilon}{2-\epsilon}\right)}{|\mathbf{r}(0)|-r_{o}},
$$

then we are guaranteed (under the conditions mentioned in the above calculations) to achieve $\Gamma\left(t_{1}\right) \leq-1+\epsilon$ at some finite time $t_{1} \leq T$.

\section{Statement of result}

Definition [8]: Given the system (1), (2) with $\Gamma$ defined by [10], we say that "motion camouflage is accessible in finite time" if for any $\epsilon>0$ there exists a time $t_{1}>0$ such that $\Gamma\left(t_{1}\right) \leq-1+\epsilon$.

Proposition: Consider the system (1), (2) with $\Gamma$ defined by (10) and control law given by (19) - 21), with the following hypotheses:

(A1) $0<\nu_{p}^{\text {low }} \leq \nu_{p} \leq \nu_{p}^{\text {high }}<\infty$, where $\nu_{p}^{\text {low }}$ and $\nu_{p}^{\text {high }}$ are constants, 
(A2) $0<\nu_{e}^{\text {low }} \leq \nu_{e} \leq \nu_{e}^{\text {high }}<\infty$, where $\nu_{e}^{\text {low }}$ and $\nu_{e}^{\text {high }}$ are constants,

(A3) $\nu_{e} / \nu_{p} \leq \nu_{\max }<1$, where $\nu_{\max }$ is constant,

(A4) $u_{e}$ and $v_{e}$ are piecewise continuous and $\sqrt{u_{e}^{2}+v_{e}^{2}}$ is bounded,

(A5) $\dot{\nu}_{e}$ and $\dot{\nu}_{p}$ are piecewise continuous, $\left|\dot{\nu}_{p}\right|<\alpha_{p}$, and $\left|\dot{\nu}_{e}\right|<\alpha_{e}$, where $\alpha_{p}$ and $\alpha_{e}$ are finite constants,

(A6) $\Gamma_{0}=\Gamma(0)<1$, and

(A7) $|\mathbf{r}(0)|>0$.

Motion camouflage is accessible in finite time using highgain feedback (i.e., by choosing $\mu>0$ sufficiently large).

Proof: Analogous to the corresponding proof in [8]. Choose $r_{o}>0$ such that $r_{o}<|\mathbf{r}(0)|$. Choose $c_{2}>0$ sufficiently large so as to satisfy 69, and choose $c_{0}$ accordingly to ensure that (53) holds for $\Gamma>-1+\epsilon$. Then defining $\mu$ according to 50 ensures that $\Gamma(T) \leq-1+\epsilon$, where $T>0$ is defined by 65 .

\section{Simulation RESUlts}

Figures 47illustrate the behavior of the three-dimensional motion camouflage system (1), (2) under control law (19) (21) for the pursuer, and various open-loop curvature controls for the evader. The speeds of the pursuer and evader are constant, and the ratio of speeds is $\nu_{e} / \nu_{p}=.9$. For each simulation, two views of the resulting three-dimensional trajectories are shown: one perpendicular to the $\mathbf{r}_{\infty}$-direction (upper plot), and one along the $\mathbf{r}_{\infty}$-direction (lower plot). In figure 4 the evader moves in a straight line (i.e., its curvature controls are identically zero). The corresponding motion camouflage trajectory for the pursuer is then also a straight line. The upper plot of figure 4 shows these straightline trajectories, along with the baselines at equally-spaced intervals of time. Recall that by definition, these baselines are parallel when the system is in a state of motion camouflage. In the lower plot of figure 4 the trajectories of the pursuer and evader overlap, and the baselines are essentially normal to the page.

In figure [5 the curvature controls for the evader are sinusoidal functions of time. Whereas in figure 4 the motion is very nearly planar (with the plane determined by the initial heading of the evader), in figure 5 the motion is seen to be truely three-dimensional. Nevertheless, the baselines are observed to be nearly parallel. In figure 6 the curvature controls for the evader are randomly varying, and similarly to figure 5 the trajectories are truly three-dimensional in character, with the baselines nearly parallel. In figure 7 the curvature controls for the evader are constant and nonzero, so that the trajectory of the evader is circular.

Although there is a brief transient period at the start of each simulation during which $\Gamma$ is driven close to -1 by the control law, this transient period is such a small fraction of the total simulation time that the transient behavior is not evident in figures 47 The effect of the gain $\mu$ on both the duration of the transient and the ultimate tolerance within which $\Gamma$ remains near -1 is illustrated for the planar

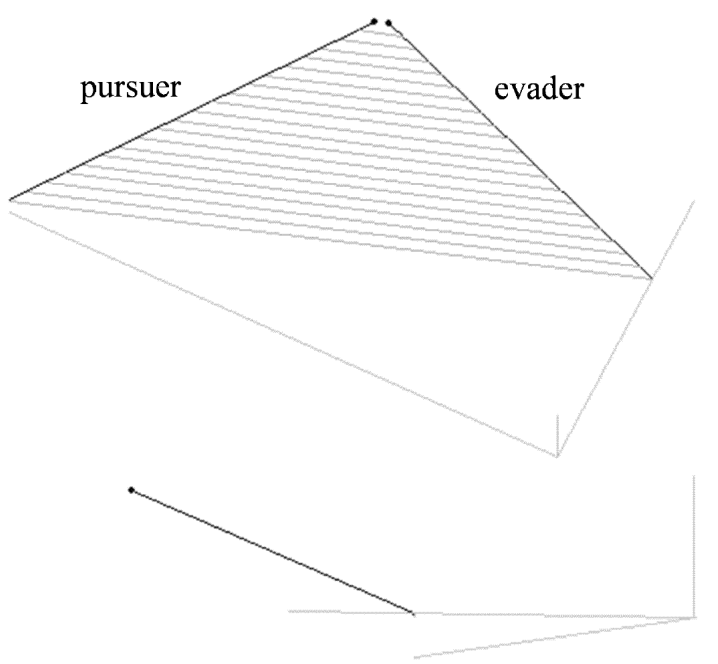

Fig. 4. Straight-line evader trajectory, and corresponding pursuer trajectory. The pursuer and evader trajectories are the dark lines (with dots at the final positions when the simulation is stopped). The light lines connecting the pursuer and evader trajectories are baselines drawn at equally spaced time intervals. The upper plot is the view perpendicular to the baseline direction, and the lower plot is the view along the baseline direction (so that the pursuer and evader trajectories overlap).

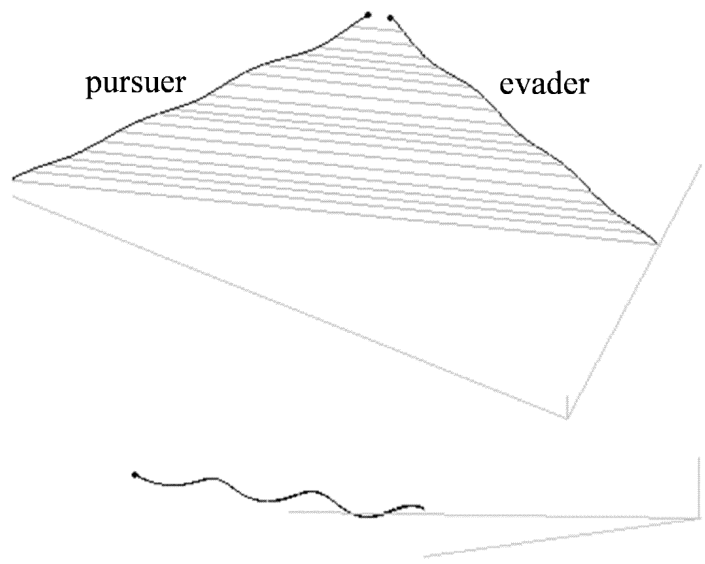

Fig. 5. Evader trajectory with sinusoidally varying curvature inputs, and corresponding pursuer trajectory.

setting in [8]. Since the bounds and estimates for the threedimensional problem are analogous to the planar problem, similar behavior is expected.

\section{Connection to missile Guidance}

For the planar setting, the connection between motion camouflage and the pure proportional navigation guidance (PPNG) law has been described in [8]. There is also a threedimensional version of the PPNG law, which has been studied in [12] and [10]. The PPNG law (by definition) produces an acceleration which is perpendicular to the velocity of the missile and proportional to the angular velocity of the line of sight (LOS) vector. If $A_{M}$ denotes the lateral acceleration of the missile, $V_{M}$ its velocity, and $\Omega_{L}$ the angular velocity of the LOS vector, then the three-dimensional PPNG law is 


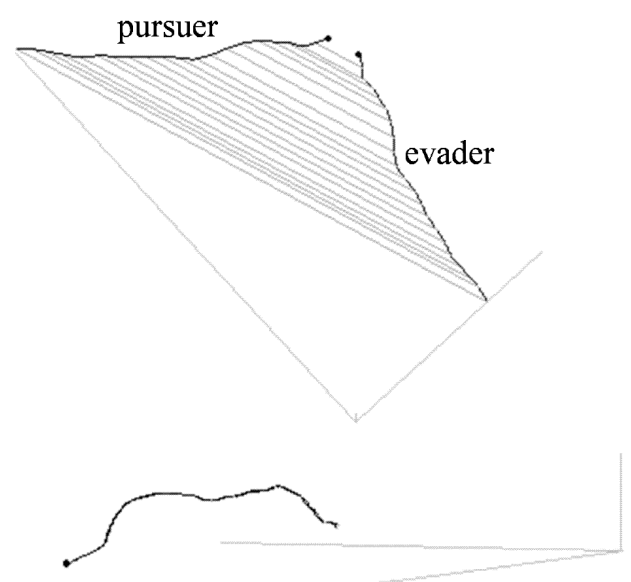

Fig. 6. Evader trajectory with randomly varying curvature inputs, and corresponding pursuer trajectory.
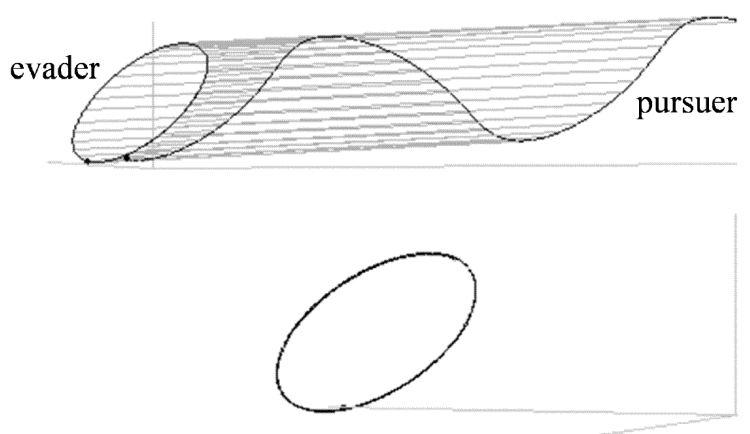

Fig. 7. Evader trajectory with constant curvature inputs (i.e., a circular trajectory), and corresponding pursuer trajectory.

given by

$$
A_{M}^{P P N G}=N\left(\Omega_{L} \times V_{M}\right),
$$

where $N>0$ is a dimensionless constant known as the navigation constant [12].

On the other hand, from equations 18, 19, 20, and 21], we observe that for the motion camouflage law, the lateral acceleration of the pursuer is

$$
\begin{aligned}
A_{M}^{M C P G} & =\mu \nu_{p}^{2} \mathbf{a}=\mu \nu_{p}^{2}\left[\mathbf{x}_{p} \times\left(\dot{\mathbf{r}} \times \frac{\mathbf{r}}{|\mathbf{r}|}\right)\right] \\
& =-\mu \nu_{p}^{2}|\mathbf{r}|\left(\mathbf{x}_{p} \times \boldsymbol{\omega}\right) .
\end{aligned}
$$

Identifying $\Omega_{L}$ with $\boldsymbol{\omega}$ and $V_{M}$ with $\nu_{p} \mathbf{x}_{p}$, we see that

$$
A_{M}^{M C P G}=\left(\mu \nu_{p}|\mathbf{r}|\right)\left(\Omega_{L} \times V_{M}\right) .
$$

To compare PPNG to MCPG, following the approach taken in the planar setting [8], we take $r_{o}$ to be a length scale for the MCPG problem, and define the dimensionless gain

$$
N^{M C P G}=\mu \nu_{p} r_{o} \text {. }
$$

Then

$$
A_{M}^{M C P G}=\left(\frac{N^{M C P G}|\mathbf{r}| / r_{o}}{N}\right) A_{M}^{P P N G} .
$$

Thus, the MCPG law uses range information to provide high gain during the initial phase of the engagement, and ramps the gain down to a lower value in the terminal phase $(|\mathbf{r}| \approx$ $\left.r_{o}\right)$. This type of gain control is plausible for echolocating bats (see [5]) which have remarkable ranging ability.

\section{DIRECTIONS FOR FUTHER WORK}

In the biological context, one direction being pursued is the interpretation of three-dimensional trajectory data taken from experiments in which a bat, Eptesicus fuscus, pursues a flying praying mantis (whose hearing organ is disabled so that its trajectory is not influenced by the presence of the bat). The hypothesis that the bat uses an MCPG strategy during the capture phase of its engagement with the mantis is currently being tested using experimental data collected in the Auditory Neuroethology Laboratory at the University of Maryland (http://www.bsos.umd.edu/psyc/batlab). This work represents part of a larger program to understand sensorymotor processing and feedback in biological model systems.

Another aspect of motion camouflage currently under study is discovering feedback laws for motion camouflage with respect to a finite point (as opposed to the point at infinity). In finite-point motion camouflage, the pursuer uses a fixed object as camouflage as it approaches the evader, and this strategy also appears to be biologically revelant. Various scenarios for motion camouflage involving teams of pursuers are also of interest, particularly in combination with formation-control laws based on gyroscopic interactions [7]. Some possible scenarios for team motion camouflage appear in [2].

\section{REFERENCES}

[1] A.J. Anderson and P.W. McOwan, "Model of a predatory stealth behavior camouflaging motion," Proc. Roy. Soc. Lond. B Vol. 270, No. 1514, pp. 489-495, 2003.

[2] A.J. Anderson and P.W. McOwan, "Motion camouflage team tactics," Evolvability \& Interaction Symposium (see http://www.des.qmul.ac.uk/aja/TEAM_MC/team_mot_cam.html, , 2003.

[3] R.L. Bishop, "There is more than one way to frame a curve," The American Mathematical Monthly, Vol. 82, No. 3, pp. 246-251, 1975.

[4] T.S. Collett and M.F. Land, "Visual control of flight behaviour in the hoverfly, Syritta pipiens," J. comp. Physiol., vol. 99, pp. 1-66, 1975.

[5] K. Ghose, T. Horiuchi, P.S. Krishnaprasad and C. Moss, "Echolocating bats use a nearly time-optimal strategy to intercept prey," PLoS Biology, to appear, 2006.

[6] P. Glendinning, "The mathematics of motion camouflage," Proc. Roy. Soc. Lond. B, Vol. 271, No. 1538, pp. 477-481, 2004.

[7] E.W. Justh and P.S. Krishnaprasad, "Natural frames and interacting particles in three dimensions," Proc. 44th IEEE Conf. Decision and Control, 2841-2846, 2005 (see also arXiv:math.OC/0503390 1).

[8] E.W. Justh and P.S. Krishnaprasad, "Steering laws for motion camouflage," preprint, 2005 arXiv:math.OC/0508023.

[9] A.K. Mizutani, J.S. Chahl, and M.V. Srinivasan, "Motion camouflage in dragonflies," Nature, Vol. 423, p. 604, 2003.

[10] J.H. Oh and I.J. Ha, "Capturability of the 3-dimensional pure PNG law," IEEE Trans. Aerospace. Electr. Syst., vol. 35, No. 2, pp. 491-503, 1999.

[11] N.A. Shneydor, Missile Guidance and Pursuit, Horwood, Chichester, 1998.

[12] S.H. Song and I.J. Ha,"A Lyapunov-like approach to performance analysis of 3-dimensional pure PNG laws," IEEE Trans. Aerospace and Electronic Systems, Vol. 30, pp. 349-358, 1994.

[13] M.V. Srinivasan and M. Davey, "Strategies for active camouflage of motion," Proc. Roy. Soc. Lond. B, Vol. 259, No. 1354, pp. 19-25, 1995.

[14] M.V. Srinivasan and S. Zhang, "Visual Motor Computations in Insects," Ann. Rev. Neurosci., Vol. 27, pp. 679-696, 2004. 